\title{
Homogeneous Esterification of Cellulose in the Mixture N-Butylpyridinium Chloride/Dimethylsulfoxide
}

\author{
Lahcen El Hamdaoui, ${ }^{1}$ Mohammed El Moussaouiti, ${ }^{1}$ and Said Gmouh ${ }^{2}$ \\ ${ }^{1}$ Laboratory of Materials Nanomaterials and Environment, Faculty of Sciences, University Mohammed V, Rabat, Morocco \\ ${ }^{2}$ National Center for Scientific Research and Technique, Rabat, Morocco
}

Correspondence should be addressed to Mohammed El Moussaouiti; elmoussa@fsr.ac.ma

Received 9 February 2016; Revised 3 May 2016; Accepted 26 May 2016

Academic Editor: Haisong Qi

Copyright (C) 2016 Lahcen El Hamdaoui et al. This is an open access article distributed under the Creative Commons Attribution License, which permits unrestricted use, distribution, and reproduction in any medium, provided the original work is properly cited.

\begin{abstract}
The present study deals with the homogeneous acylation of cellulose with $p$-nitrobenzoyl chloride in a reaction medium composed of a mixture of 1-butylpyridinium chloride and dimethylsulfoxide (BPyCl/DMSO), in the presence of different bases and under mild conditions. The preparation of cellulose $p$-nitrobenzoate depending on the reaction conditions, the influences of reaction parameters such as the base type, and the types of cellulose (kraft and microcrystalline) on the products were investigated. Cellulose $p$-nitrobenzoate with a degree of substitution (DS) in the range from 0.12 to 1.5 was accessible, with a low excess of reagent and for a short reaction time. The cellulose esters were characterized by ${ }^{1} \mathrm{H}-\mathrm{NMR},{ }^{13} \mathrm{C}$ NMR, and FT-IR spectroscopy, thermogravimetric analysis (TGA), scanning electron microscopy (SEM), and solubility tests.
\end{abstract}

\section{Introduction}

Cellulose is the most abundant natural polymer; its derivative products have many important applications in fiber, membrane, paper, polymer, and paint industries [1-3]. The structure of cellulose consists of both crystalline and amorphous regions, and this construction makes it difficult to be dissolved in normal solvents. Cellulose modification, under homogeneous conditions, particularly esterification, has received increased attention during the last several decades, aiming for better conversion and distribution. The mostly applied solvents in this modification process are N,N-dimethylacetamide/lithium chloride (DMAc/LiCl) [4], a mixture of dimethylsulfoxide (DMSO) and tetrabutylammonium fluoride trihydrate $\left(\mathrm{TBAF} \times 3 \mathrm{H}_{2} \mathrm{O}\right)$ [5], various ionic liquids (ILs), namely, 1-N-butyl-3-methylimidazolium chloride $([\mathrm{Bmim}] \mathrm{Cl})$, and 1-allyl-3-methylimidazolium chloride $([\mathrm{Amim}] \mathrm{Cl})[6,7]$. The 3-methyl-N-butyl-pyridinium chloride and benzyldimethyltetradecylammonium chloride were also investigated as a new kind of solvents for cellulose. The ILs used have the ability to dissolve cellulose with a degree of polymerization (DP) in the range from 290 to 1200 to a very high concentration $[8,9]$. Homogeneous esterification of cellulose with acyl-1H-benzotriazole and 4-(4,6-dimethoxy1,3,5-triazin-2-yl)-4-methyl-morpholinium chloride and $\mathrm{N}$-alkyl-2-halopyridinium salts as activating agents in DMSO/TBAF was carried out. It has been found that N,Ncarbonyldiimidazole (CDI) is most effective regarding the degree of substitution (DS) values [10]. In order to expand the scope of ILs used for cellulose esterification and explore the possibility of using nonimidazolium type of ILs, the dissolution and homogeneous acetylation of cellulose into the novel protic IL 1,5-diazabicyclo[4.3.0]non-5-enium acetate ([DBNH][OAc]) with a dispersing agent such as acetone, acetonitrile, DBN, or DMSO under mild conditions were reported [11]. Also, acetylation of xylan in [DBNH][OAc], followed by acetic anhydride/1,5-diazabicyclo[4.3.0]non-5ene (Ac2O/DBN) and no cosolvent added, was investigated [12]. Room-temperature ionic liquids, being considered as desirable green solvents for a wide range of separation and as reaction media for processes including catalysis, have recently received significant attention $[13,14]$. Synthesis and structural characterizations of p-bromobenzoate and p-phenylbenzoate of kraft and microcrystalline cellulose in 
[Bmim] $\mathrm{Cl}$ were carried out [15]. ILs are almost nonvolatile, nonflammable, thermally stable, and reusable "green" solvents. Particularly, used as reaction media, ILs have several advantages such as enhancement of reaction rates, improvement of selectivity and yields, or ease of recycling catalysts [16, 17]. For example, acetylation of alcohols and saccharides was conducted in a dicyanamide based ionic liquid [18]. Because of their unique properties, ILs are currently being explored as environmentally benign solvent substitutes for traditional volatile organic solvents in a variety of applications $[19,20]$.

In the present study, we describe the homogeneous esterification of cellulose in $\mathrm{BPyCl} / \mathrm{DMSO}$ solvent system, applying $p$-nitrobenzoyl chloride as acylation agent. The cellulose $p$ nitrobenzoate obtained was characterized by means of FTIR, NMR spectroscopy, and solubility tests. Thermogravimetric analysis (TGA) was used for degradation and char content information of the modified celluloses by measuring the change in mass as a function of temperature. The scanning electron microscopy (SEM) was used to monitor the morphology changes. The main objective of this work is to develop novel sustainable ionic liquid-based systems (IL/cosolvent) with the capability to dissolve cellulose of high molecular weight and to carry out the homogeneous esterification of cellulose. In order to develop an efficient, commercially and environmentally viable IL-based process, recycling of ILs is of crucial importance.

\section{Experimental}

2.1. Materials. Commercial microcrystalline cellulose with a degree of polymerization (DP $\approx 200$ ) (Powder Aldrich Chemical) and bleached kraft cellulose (DP $\approx 1200$ ) provided by Cellulose of Morocco Company were used as initial cellulose. The cellulose was dried for $6 \mathrm{~h}$ at $60^{\circ} \mathrm{C}$ until constant weight before use. All other standard chemicals were purchased from ACROS chemicals or Aldrich chemical and used as is received without further purification. The IL 1-butylpyridinium chloride (BPyCl) was synthesized in accordance with the literature [6].

2.2. Synthesis of 1-Butylpyridinium Chloride. The pyridine (8, $21 \mathrm{~g})$ and butane chloride $(20 \mathrm{~g})$ at a molar ratio $1: 2$ were added to a round-bottom flask fitted with a reflux condenser for $48 \mathrm{~h}$ at $80^{\circ} \mathrm{C}$ with stirring. The solid is washed with ethyl acetate $(3 \times 20 \mathrm{~mL})$ and dried under vacuum. The obtained product $(\mathrm{BPyCl})$ had a solid bright white color, yield $84 \%$. Synthesis of 1-butylpyridinium chloride is shown in Scheme 1.

2.3. Dissolution of Cellulose in $(B P y C l) / D M S O$. Cellulose $(2 \mathrm{w} \%)$ was suspended in a mixture of 1-butylpyridinium chloride/DMSO $(70 \mathrm{w} / 30 \mathrm{w})$ in a round-bottom flask. This mixture was stirred with a magnetic stirrer at $80^{\circ} \mathrm{C}$ for a maximum of $12 \mathrm{~h}$. The solubility of cellulose in $\mathrm{BPyCl} / \mathrm{DMSO}$ mixture was checked visually.

2.4. Synthesis of Cellulose p-Nitrobenzoate (General Procedure). A mixture of cellulose ( $2 \mathrm{w} \%)$, 1-butylpyridinium chloride/dimethylsulfoxide $(70 \mathrm{w} / 30 \mathrm{w}), \quad p$-nitrobenzoyl

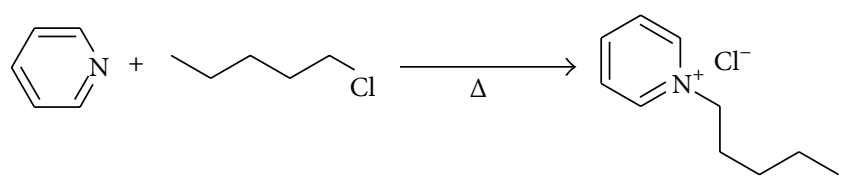

SCHEME 1: Reaction scheme for the synthesis of 1-butylpyridinium chloride.

chloride ( 5 mol equivalent), and the base ( 5 mol equivalent) was heated in an oil bath to $80^{\circ} \mathrm{C}$ and kept at this temperature for $1.30 \mathrm{~h}$. The reaction mixture was precipitated in $100 \mathrm{~mL}$ isopropanol. The obtained cellulose $p$-nitrobenzoate was filtered and washed several times with methanol and then dried at $40^{\circ} \mathrm{C}$ in vacuum.

IR (KBr): $\quad 3378.66 \mathrm{~cm}^{-1} v(\mathrm{OH}), \quad 2914 \mathrm{~cm}^{-1} v(\mathrm{CH}$ cellulose), $\quad 1730 \mathrm{~cm}^{-1} v(\mathrm{C}=\mathrm{O}$ ester $), \quad 1628 \mathrm{~cm}^{-1}$ $v(\mathrm{C}=\mathrm{C}), \quad 1260 \mathrm{~cm}^{-1} v(\mathrm{C}-\mathrm{O}), \quad 1100 \mathrm{~cm}^{-1} v(\mathrm{C}-\mathrm{O}-\mathrm{C})$, $1528 \mathrm{~cm}^{-1} v\left(\mathrm{NO}_{2}\right)$, and $850 \mathrm{~cm}^{-1} v(\mathrm{CN})$.

${ }^{13} \mathrm{C}-\mathrm{NMR}\left(\mathrm{DMSO}-\mathrm{d}_{6}\right): \delta=60.5(\mathrm{C}-6), \delta=73-75.3$

$(\mathrm{C}-5,3,2), \delta=83.5(\mathrm{C}-4), \delta=103(\mathrm{C}-1), \delta=130$

$(\mathrm{C}-10), \delta=133.5(\mathrm{C}-8,9), \delta=152(\mathrm{C}-11)$, and $\delta=$ $170 \mathrm{ppm}(\mathrm{C}-7)$.

${ }^{1} \mathrm{H}$-NMR (DMSO- $\mathrm{d}_{6}$ ): cellulose $p$-nitrobenzoate: $\delta=$ $7.3-8$ (phenyl protons, $4 \mathrm{H}$ ) and $\delta=3.3-5$ (protons of kraft cellulose, $7 \mathrm{H}$ ).

${ }^{1} \mathrm{H}-\mathrm{NMR}$ is usually convenient and is widely used for DS determination of cellulose derivatives. The peaks for $p$ nitrobenzoyl at around 7.3-8.2 ppm strongly overlap with signals for the protons of the cellulose AGU in the range 3.3$5 \mathrm{ppm}$. The partial DS value of the acetyl moiety among the three $\mathrm{OH}$ groups can be inferred from the integration of the ${ }^{13} \mathrm{C}$ NMR spectra of a solution of cellulose ester in DMSO- $\mathrm{d}_{6}$.

The DS of cellulose ester was calculated from the ${ }^{1} \mathrm{H}$ NMR spectra [21] by equation

$$
\mathrm{DS}=\frac{7 I_{\text {phenyl }}}{4 I_{\mathrm{AGU}}},
$$

where $I_{\text {phenyl }}$ is the peak integral of phenyl protons at 7.3$8.2 \mathrm{ppm}$ and $I_{\mathrm{AGU}}$ is the peak integral of protons of anhydroglucose unit at 3.3-5 $\mathrm{ppm}$.

2.5. Spectroscopic Measurements. The chemical structure of cellulose and cellulose ester samples was analyzed by FT-IR, ${ }^{13} \mathrm{C}$ NMR, and ${ }^{1} \mathrm{H}-\mathrm{NMR}$ spectroscopy techniques.

FT-IR. A mass of $1 \mathrm{mg}$ of the substrate is crushed and mixed with $50 \mathrm{mg}$ of potassium bromide $\mathrm{KBr}(99 \%)$. The obtained powder is then pelletized under a pressure of 6 bars. The spectra were recorded on Vertex 70 spectrometer at room temperature. All spectra were recorded by accumulating 64 scans at a resolution of $4 \mathrm{~cm}^{-1}$ in the range of 4000 to $400 \mathrm{~cm}^{-1}$.

NMR spectra were acquired on a Bruker Advance $300 \mathrm{MHz}$ spectrometer with 16 scans for ${ }^{1} \mathrm{H}$-NMR and up to 81920 scans for ${ }^{13} \mathrm{C}$ NMR at $60^{\circ} \mathrm{C}$ in deuterated dimethylsulfoxide (DMSO- $\mathrm{d}_{6}$ ) and a sample concentration of $60 \mathrm{mg} / \mathrm{mL}$. 


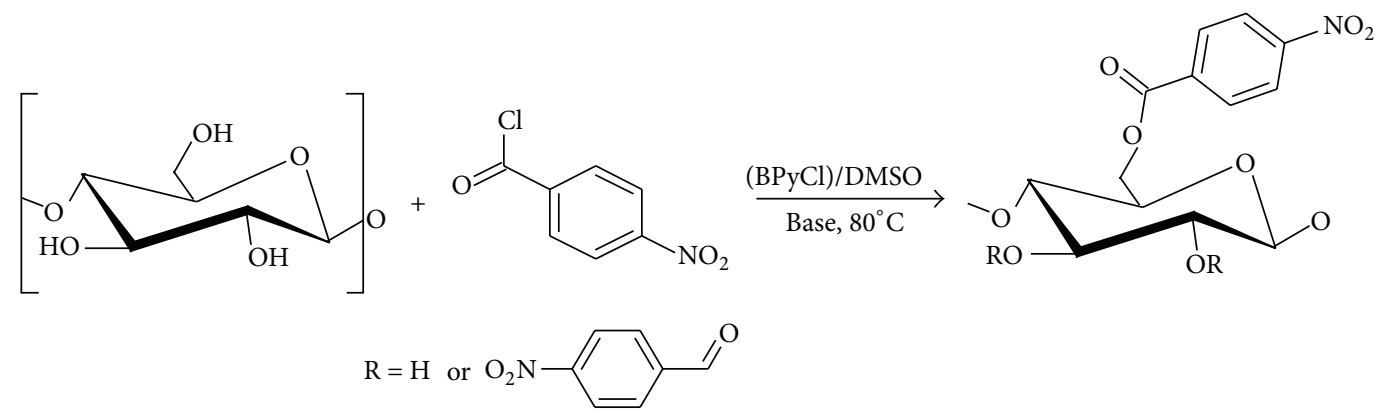

SCHEme 2: General synthesis of cellulose $p$-nitrobenzoate.

2.6. Thermal Analysis. The analysis of the kraft cellulose and grafted cellulose samples was performed using thermogravimetric analysis (TGA) on a simultaneous thermal analyzer SETARAM LABSYS EVO (1F). The apparatus was continually flushed with nitrogen. The 10 to $13 \mathrm{mg}$ samples were heated from room temperature up to $600^{\circ} \mathrm{C}$ in aluminum pan with a constant heating rate of $10^{\circ} \mathrm{C} / \mathrm{min}$. Three samples were used to characterize each material.

2.7. Morphological Surface Analysis. SEM analyses were performed with a FEI Quanta 200 microscope to observe the morphology of cellulose and cellulose derivatives. Before SEM analysis, the dried cellulose samples were coated with a carbon layer to increase their conductivity. All samples were examined using an accelerating voltage of $30 \mathrm{kV}$.

2.8. Solubility. The solubility of cellulose ester at room temperature was checked in the following solvents: N,Ndimethylacetamide (DMAc), dimethylsulfoxide (DMSO), dimethylformamide (DMF), and water, considering a concentration of $0.02 \mathrm{~g} / \mathrm{mL}$ solvent.

\section{Results and Discussion}

3.1. Synthesis of Cellulose p-Nitrobenzoate. Recently, the solubility of cellulose in various ionic liquids has been reported $[6,22-24]$. Even better cellulose solvents than the ILs with chloride as their anion are ILs with acetate as an anion. The homogeneous dissolution of cellulose in ionic liquid 1butylpyridinium chloride ( $\mathrm{BPyCl}$ ) with cosolvent dimethylsulfoxide (DMSO) can be used to perform functionalization reactions to introduce functionalities to the cellulose backbone. As a model reaction, the esterification with $p$ nitrobenzoyl chloride was chosen; since it is a regioselective protection reaction for the more reactive primary hydroxyl group. The cellulose $p$-nitrobenzoate could be used as raw material for the preparation of other cellulose derivatives by modification of nitro groups $\left(\mathrm{NO}_{2}\right)$. The general reaction scheme of the nitrobenzoylation is shown in Scheme 2.

\subsection{Structure Characterization}

3.2.1. FT-IR Spectroscopy. FT-IR spectra of the kraft cellulose, the regenerated kraft cellulose, and the kraft cellulose $p$-nitrobenzoate sample from $(\mathrm{BPyCl}) / \mathrm{DMSO}$ are shown in

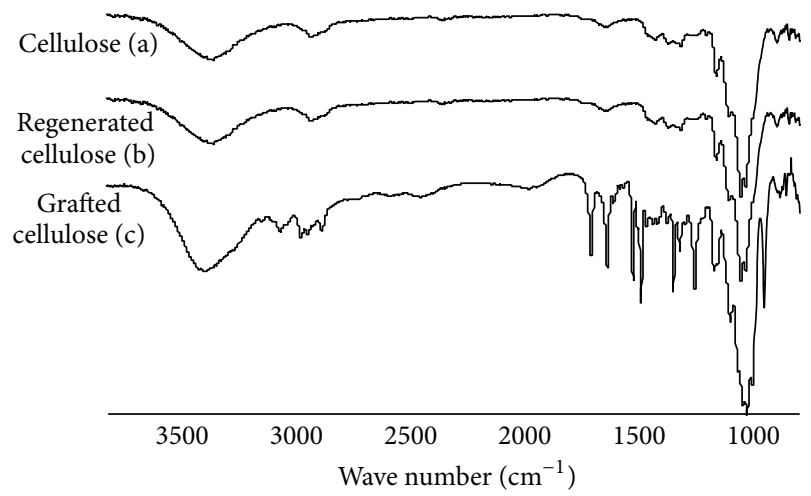

FIGURE 1: IR spectra of kraft cellulose (a), regenerated cellulose, (b) and grafted cellulose (c).

Figure 1. It can be seen that spectra (a) and (b) are quite similar and no new peaks appear in the regenerated sample, indicating no chemical reaction occurred during the cellulose dissolution and regeneration processes. In other words, $(\mathrm{BPyCl}) / \mathrm{DMSO}$ is a nonderivatizing solvent for cellulose. Spectrum (c) provides a clear evidence of acetylation by showing the presence of some important peaks at $1730 \mathrm{~cm}^{-1}$ for $(\mathrm{C}=\mathrm{O})$ stretching ester, $1260 \mathrm{~cm}^{-1}$ for stretching of $(\mathrm{C}$ $\mathrm{O}), 3053 \mathrm{~cm}^{-1}$ for aromatic ring $(\mathrm{C}-\mathrm{H})$ stretching, $1628 \mathrm{~cm}^{-1}$ for aromatic $(\mathrm{C}=\mathrm{C})$ stretching, $3378 \mathrm{~cm}^{-1}$ for group $(\mathrm{OH})$ stretching of cellulose, $2914 \mathrm{~cm}^{-1}$ for $(\mathrm{C}-\mathrm{H})$ cellulose, and $1100 \mathrm{~cm}^{-1}$ for (C-O-C) asymmetric stretching and ring asymmetric stretching for cellulose. The strong absorption peaks at $1528 \mathrm{~cm}^{-1}$ and $850 \mathrm{~cm}^{-1}$ correspond, respectively, to $\mathrm{NO}_{2}$ symmetric stretching and $(\mathrm{C}-\mathrm{N})$ stretching. These differential FT-IR results confirmed the reactions of esterification of cellulose by $p$-nitrobenzoyl chloride in a mixture (BPyCl)/DMSO.

The results of the homogeneous reaction of cellulose with p-nitrobenzoyl chloride (5 eq.) in a mixture (BPyCl)/DMSO with $2 \%(\mathrm{w} / \mathrm{w})$ cellulose were allowed to react at $80^{\circ} \mathrm{C}$. Reaction conditions, DS values of the obtained cellulose esters, and their solubility are summarized in Table 1.

Initial attempts at the homogeneous esterification of kraft and microcrystalline cellulose in (BPyCl)/DMSO mixture were carried out at $80^{\circ} \mathrm{C}$ for $12 \mathrm{~h}$ without any base. The obtained outcome was a black mixture, which indicates a 
TABLE 1: Reaction conditions, DS, and solubility for obtained cellulose $p$-nitrobenzoate.

\begin{tabular}{|c|c|c|c|c|c|c|c|c|}
\hline \multirow{2}{*}{ Entry } & \multirow{2}{*}{ Cellulose type } & \multirow{2}{*}{ Base } & \multirow{2}{*}{ Reaction time (h) } & \multirow{2}{*}{$\mathrm{DS}^{\mathrm{a}}$} & \multicolumn{4}{|c|}{ Solubility } \\
\hline & & & & & DMSO & $\mathrm{DMF}$ & DMAc & $\mathrm{H}_{2} \mathrm{O}$ \\
\hline 1 & Microcrystalline & DMAP & 1.30 & 0.4 & + & - & - & - \\
\hline 2 & Microcrystalline & Pyridine & 1.30 & 0.23 & + & - & - & - \\
\hline 3 & Microcrystalline & Triethylamine & 1.30 & 0.19 & + & - & - & - \\
\hline 4 & Microcrystalline & DMAP & 3.0 & 1.50 & + & + & + & - \\
\hline 5 & Kraft & DMAP & 1.30 & 0.35 & + & - & & - \\
\hline 6 & Kraft & Pyridine & 1.30 & 0.19 & + & - & - & - \\
\hline 7 & Kraft & Triethylamine & 1.30 & 0.12 & + & - & - & - \\
\hline 8 & Kraft & DMAP & 3.0 & 1.42 & + & + & + & - \\
\hline
\end{tabular}

degradation of the cellulose. No precipitated product resulted from the addition of isopropanol, ethanol, or methanol to the black mixture. This confirms again that the cellulose has been degraded. It is assumed that the black color is caused by a combination of the released hydrogen chloride and the decomposition products of cellulose.

On another occasion, the esterification was performed on $(\mathrm{BPyCl}) / \mathrm{DMSO}$ mixture, using tertiary bases such as triethylamine, pyridine, and 4-dimethylaminopyridine (DMAP) and reducing the reaction time to $3 \mathrm{~h}$ with the intention of capturing the released hydrogen chloride and suppressing the degradation of cellulose. The finding was that the brown color replaced the black one.

The effect of reaction parameters such as reaction time and base type on the synthesis of cellulose $p$-nitrobenzoate in a mixture $(\mathrm{BPyCl}) / \mathrm{DMSO}$ has been studied. The DS of the products increases as reaction time prolongs (entries 1 , 4 and 5,8). Table 1 shows that, under conditions of $80^{\circ} \mathrm{C}$ and DMAP (5 eq.), the DS of the acylated product reaches 0.4 for microcrystalline cellulose and 0.35 for kraft cellulose within a period of $1.30 \mathrm{~h}$, whereas it increases to 1.5 and 1.42 within $3 \mathrm{~h}$ for the microcrystalline cellulose and $\mathrm{kraft}$ cellulose, respectively.

Further investigation on the influence of the bases was carried out (entries 1, 2, and 3) keeping the ratio of $p$-nitrobenzoyl chloride to cellulose at $5 \mathrm{~mol}$ equivalent, and adding $5 \mathrm{~mol}$ equivalent of various bases. The DS of the obtained cellulose $p$-nitrobenzoate has reached 0.19 , 0.23 , and 0.4 for triethylamine, pyridine, and DMAP, respectively. It is clear that, by using DMAP and pyridine, the reactivity of acylation systems increases. This might be due to the stabilization of the acylpyridinium ion, which plays an important role in the catalytic cycle and results in an increasing rate of the reaction.

The results presented in Table 1 also show that the DS of kraft cellulose is lower than that of the MCC, and the degree of substitution of obtained products increased with decrease in the degree of polymerization. This is due to the fact that, with the decrease in molecular weight, more numbers of hydroxyl groups become available to undergo esterification reaction giving higher DS values in comparison to high molecular mass cellulose. These results suggest that it is possible to control the DS value of cellulose $p$-nitrobenzoate by controlling reaction time and the choice of the used base. The three hydroxyl groups at the C2, C3, and C6 positions exhibit various reaction activities. In fact, Figure 2 shows a ${ }^{13} \mathrm{C}$ NMR spectrum of sample (4) with a DS of 1.5 in which the signal at $170.2 \mathrm{ppm}$ was attributed to the carbonyl carbon at C-6, the signal at $167.4 \mathrm{ppm}$ to carbon at C-2, and the signal at $166.2 \mathrm{ppm}$ to carbon at C-3. Obviously, the nitrobenzoylation reaction is favored at $\mathrm{C}-6$, and the order of reactivity is C6$\mathrm{OH}>\mathrm{C} 2-\mathrm{OH}>\mathrm{C} 3-\mathrm{OH}$. This result is similar to that observed in [Amim]Cl solution [25].

3.3. Solubility. In this work, the solubility of synthesized cellulose $p$-nitrobenzoate in a mixture ionic liquid/cosolvent (BPyCl/DMSO), DMAc, DMF, DMSO, and water was investigated, and the results are summarized in Table 1. All esters products are readily soluble in DMSO and insoluble in the water, but the solubility of the ester in dimethylformamide and N,N-dimethylacetamide depends greatly on the total DS value.

3.4. Thermogravimetric Analysis. During the TGA study of the thermal behavior of kraft cellulose, $p$-nitrobenzoyl chloride, and cellulose ester (DS $\approx 1$ ), the temperature was increased from 25 to $600^{\circ} \mathrm{C}$, according to a heating speed of $10^{\circ} \mathrm{C} / \mathrm{min}$, and maintained constant at $95^{\circ} \mathrm{C}$ for $15 \mathrm{~min}$ to remove adsorbed water. The results of the analysis are summarized in Figure 3. The main thermal characteristics from the TGA-DTG curves, such as the temperature of the beginning of the degradation $T_{0}$ corresponding to $1 \%$ mass loss, the temperature of maximum mass loss $T_{\max }$, and the percentage of carbon residue $(\mathrm{CR} \%)$ at $T_{\max }$ and at $400^{\circ} \mathrm{C}$, are given in Table 2.

Results of the TGA and the DTG represented in Figure 3 show that kraft cellulose (a) degrades between 280 and $360^{\circ} \mathrm{C}$ and the speed of mass loss has a peak around $350^{\circ} \mathrm{C}$ (mass loss 42\%). These results are consistent with those of the literature [26-28]. The TGA and DTG spectra for $p$ nitrobenzoyl chloride (b) can be decomposed into two zones. The first one is located between 110 and $280^{\circ} \mathrm{C}$ with a DTG peak centered at $254^{\circ} \mathrm{C}$ (mass loss $35 \%$ ). The second zone, which takes place between 280 and $375^{\circ} \mathrm{C}$ with a maximal 

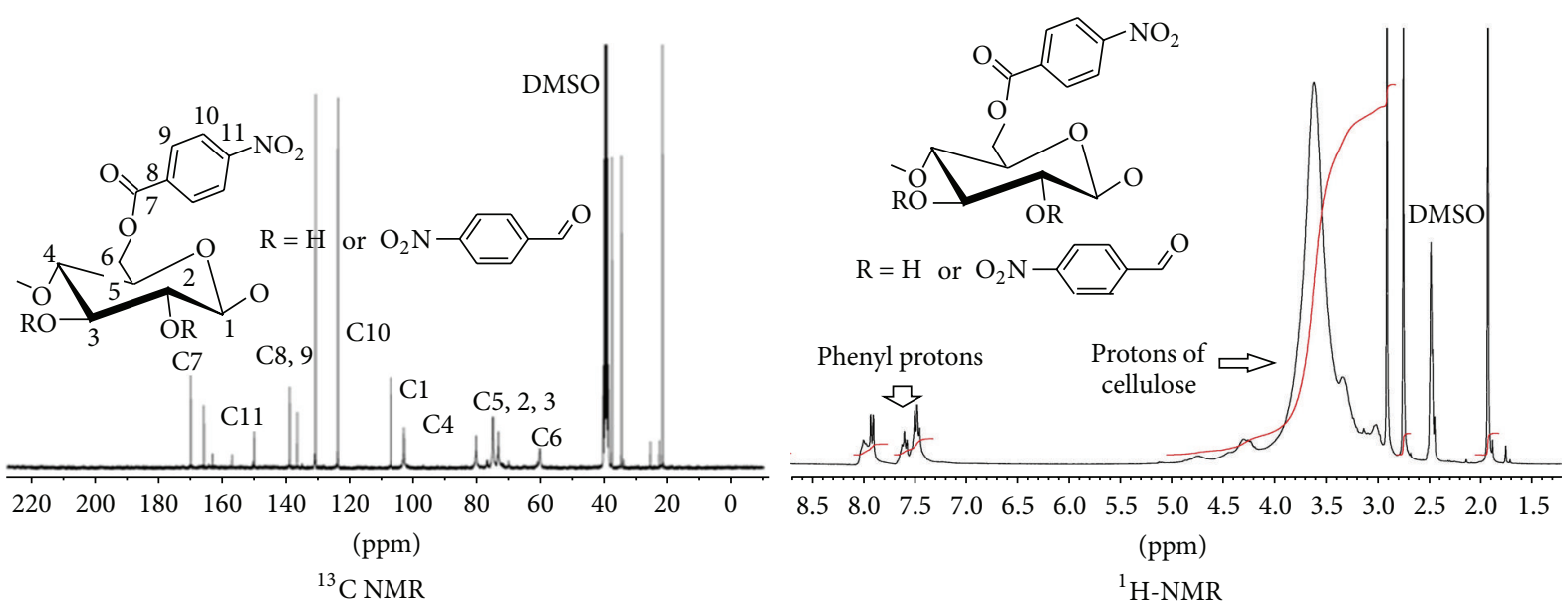

FIGURE 2: NMR spectra of kraft cellulose $p$-nitrobenzoate.

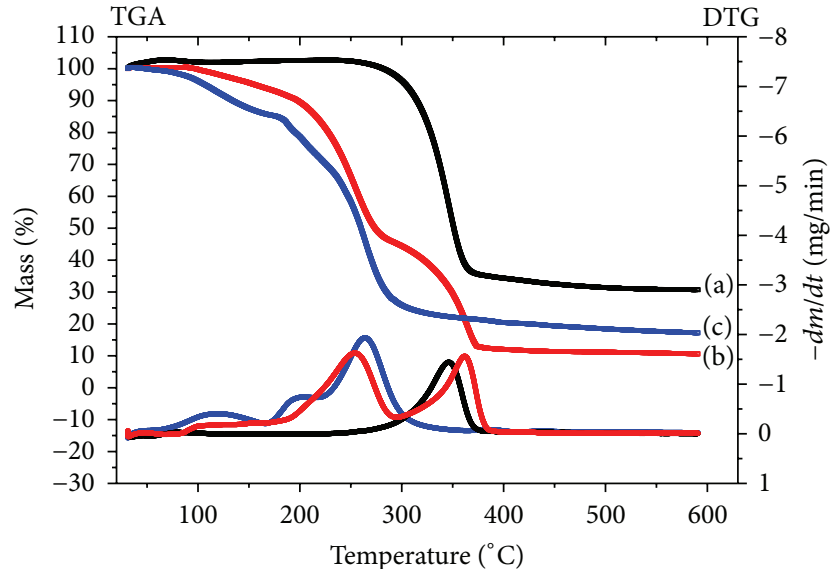

FIGURE 3: Thermogravimetric analysis TGA-DTG for kraft cellulose (a), p-nitrobenzoyl chloride (b), and cellulose $p$-nitrobenzoate (c).

TABLE 2: Thermal characteristics of samples kraft cellulose (a), $p$ nitrobenzoyl chloride (b), and cellulose p-nitrobenzoate (c).

\begin{tabular}{lcccc}
\hline Samples & $T_{0}\left({ }^{\circ} \mathrm{C}\right)$ & $T_{\max }\left({ }^{\circ} \mathrm{C}\right)$ & $\begin{array}{c}\mathrm{CR}^{\mathrm{a}}(\%) \\
\left(\text { at } T_{\max }\right)\end{array}$ & $\begin{array}{c}\mathrm{CR}^{\mathrm{b}}(\%) \\
\left(\text { at } 400^{\circ} \mathrm{C}\right)\end{array}$ \\
\hline (a) & 280 & 350 & 58 & 34 \\
(b) & 100 & $254-360$ & $65-24$ & 12 \\
(c) & 80 & 264 & 45 & 20 \\
\hline
\end{tabular}

$T_{0}$ : temperature of the beginning of the degradation to $1 \%$ mass loss.

$T_{\text {max }}$ : temperature of maximum mass loss.

$\mathrm{CR}^{\mathrm{a}}$ (\%): percentage of carbon residue at $T_{\max }$.

$\mathrm{CR}^{\mathrm{b}}$ (\%): percentage of carbon residue at $400^{\circ} \mathrm{C}$.

mass loss speed at $360^{\circ} \mathrm{C}$ (mass loss $76 \%$ ), is related to slower decomposition processes. The TGA and DTG curves of the cellulose ester (c) are fundamentally different from those of the other various constituents. DTG peaks observed for the p-nitrobenzoyl chloride $\left(254^{\circ} \mathrm{C}\right.$ and $\left.360^{\circ} \mathrm{C}\right)$ and the cellulose $\left(350^{\circ} \mathrm{C}\right)$ disappeared. DTG peak of the final compound presents only one main peak at $264^{\circ} \mathrm{C}$ (mass loss 55\%), at a lower temperature than the main peak of decomposition of the cellulose $\left(350^{\circ} \mathrm{C}\right)$ and at a temperature between the two peaks of the $p$-nitrobenzoyl chloride. DTG peak of the cellulose ester becomes wider and shifts to lower temperature, and the amount of carbon residue at $400^{\circ} \mathrm{C}$ and at $T_{\max }$ decreases by $\sim 14 \%$ and is observed as compared to the unmodified kraft cellulose. These results showed decreased thermal stability for cellulose $p$-nitrobenzoate due to a decrease in crystallinity of cellulose modified by esterification and to the in situ formation of nitrogen containing $\mathrm{NO}_{2}$, which accelerate cellulose decomposition. Even if the interpretation is difficult, it can be concluded that there is a significant chemical modification of initial substrates and thus the formation of a chemical bond between these molecules, confirming the occurrence of grafting.

3.5. Morphological Surface Analysis. SEM analysis was mainly employed to determine the surface properties of the samples. Figure 4 shows the SEM micrograph illustrating the morphology of microcrystalline cellulose (a), kraft cellulose $(d)$, and their corresponding regenerated $(b, e)$ and grafted (c, f) celluloses.

The analysis of the SEM pictures reveals a number of things: First, the microcrystalline cellulose (MCC) is mainly composed of rod-like and platelet-like cellulose micro-fibrils, shaped into a spherical agglomeration. Second, the kraft cellulose has a three-dimensional structure made of nanometric fibers. Before their treatment, these fibrils are identifiable almost one by one, but this distinction becomes very difficult after the regeneration and esterification treatments. Initially and before treatments the fibrils exist at an elementary level with a variable size within 3-20 $\mathrm{nm}$, depending on the source of cellulose (a, d) [29]. Third, under the electron microscopic examination, the regenerated celluloses obtained from the microcrystalline (b) and kraft (e) cellulose are nonporous, with a plain texture and a surface structure that is smooth, compact, and homogeneous. Fourth, the morphology of the cellulose nitrobenzoate derivative (DS $\approx 1$ ) is clearly different from the morphologies of microcrystalline and kraft celluloses, which indicates successful grafting. 


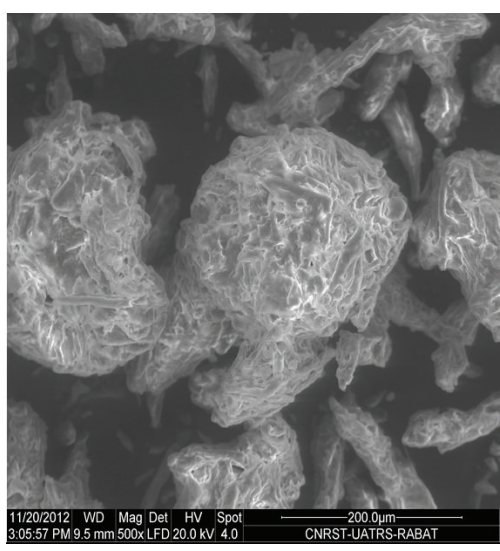

(a)

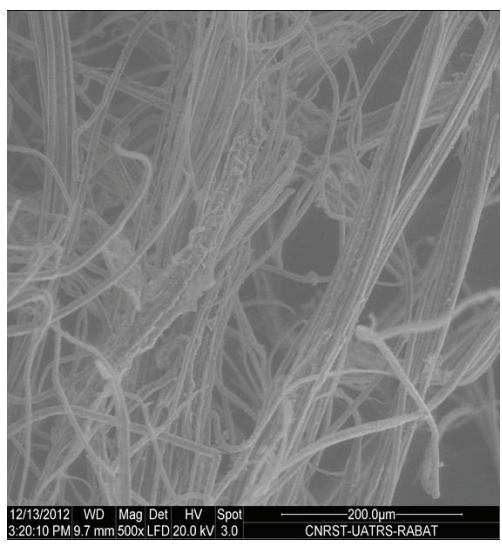

(d)

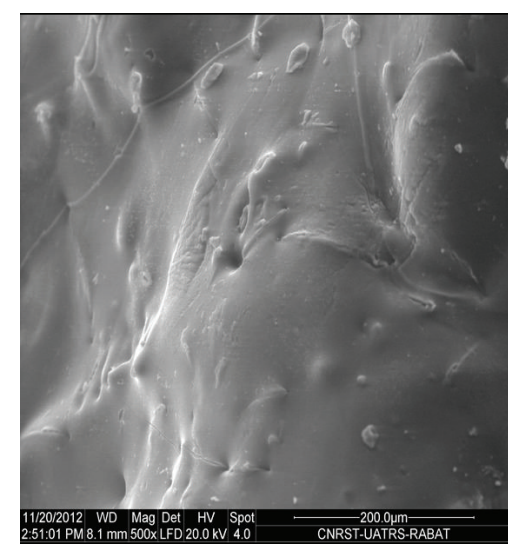

(b)

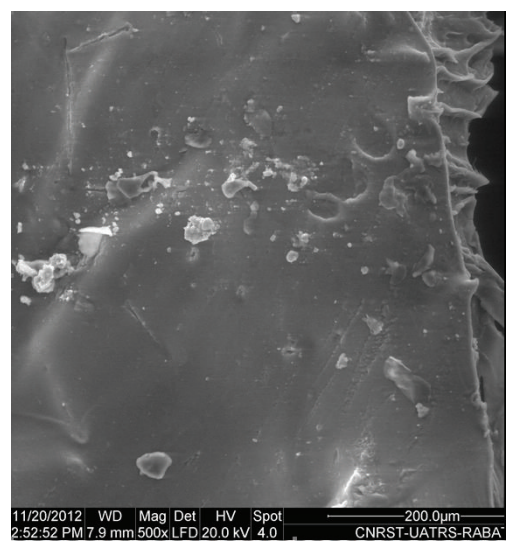

(e)

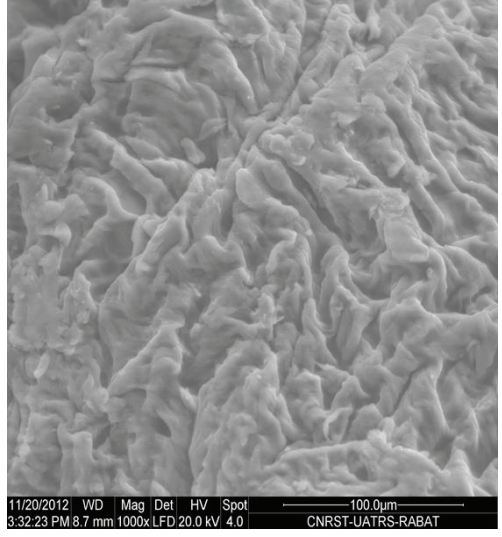

(c)

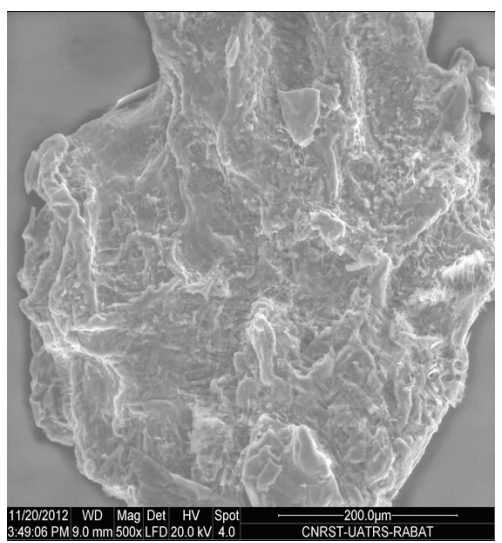

(f)

FIGURE 4: SEM micrographs of microcrystalline cellulose (a), regenerated microcrystalline cellulose (b), grafted microcrystalline cellulose (c), kraft cellulose (d), regenerated kraft cellulose (e), and grafted kraft cellulose (f).

\section{Conclusion}

The results show that $(\mathrm{BPyCl}) / \mathrm{DMSO}$ is a direct solvent to dissolve cellulose. In general, it was found that cellulose dissolved very well in ( $\mathrm{BPyCl}) / \mathrm{DMSO}$ and the solubility can reach $5 \mathrm{wt} \%$ at $80^{\circ} \mathrm{C}$. Mixture of IL with a cosolvent was successfully applied for the synthesis of cellulose $p$-nitrobenzoate by reacting cellulose with $p$-nitrobenzoyl chloride in the presence of base under mild conditions. The resulted compounds were characterized by spectroscopy techniques (FTIR, NMR). The DS values were obtained by ${ }^{1} \mathrm{H}-\mathrm{NMR}$ technique.

It was shown that cellulose esters with different DS can be obtained by varying the reaction time in a completely homogeneous synthesis. The products dissolve in some organic solvents such as DMSO, DMAc, and DMF, depending on their DS values. The detailed studies about the interaction cellulose/solvent are still under investigation. The thermal behaviors were investigated by thermogravimetric analysis (TGA) under nitrogen. The results indicated some differences between thermal degradation of cellulose and cellulose $p$-nitrobenzoate. The cellulose derivatives exhibited less heat resistance compared to cellulose, meaning that cellulose is a thermally more stable material than cellulose p-nitrobenzoate. Surface morphology of grafting cellulose is clearly different from the surface morphologies of cellulose, which proves the grafting success.

\section{Competing Interests}

The authors declare that they have no competing interests.

\section{References}

[1] T. Heinze, "New ionic polymers by cellulose functionalization," Macromolecular Chemistry and Physics, vol. 199, no. 11, pp. 23412364, 1998.

[2] T. Heinze and T. Liebert, Progress in Polymer Science, vol. 66, p. 1689,2001

[3] K. J. Edgar, C. M. Buchanan, J. S. Debenham et al., "Advances in cellulose ester performance and application," Progress in Polymer Science, vol. 26, no. 9, pp. 1605-1688, 2001.

[4] C. L. McCormick, P. A. Callais, and B. H. Hutchinson Jr., "Solution studies of cellulose in lithium chloride and N,N-dimethylacetamide," Macromolecules, vol. 18, no. 12, pp. 2394-2401, 1985.

[5] T. Heinze, R. Dicke, A. Koschella, A. H. Kull, E.-A. Klohr, and W. Koch, "Effective preparation of cellulose derivatives in a 
new simple cellulose solvent," Macromolecular Chemistry and Physics, vol. 201, no. 6, pp. 627-631, 2000.

[6] R. P. Swatloski, S. K. Spear, J. D. Holbrey, and R. D. Rogers, "Dissolution of cellose with ionic liquids," Journal of the American Chemical Society, vol. 124, no. 18, pp. 4974-4975, 2002.

[7] Q. Ren, J. Wu, J. Zhang, J. S. He, and M. L. Guo, "Synthesis of 1-allyl,3-methylimidazolium-based room-temperature ionic liquid and preliminary study of its dissolving cellulose," Acta Polymerica Sinica, vol. 1, no. 3, pp. 448-451, 2003.

[8] S. Barthel and T. Heinze, "Acylation and carbanilation of cellulose in ionic liquids," Green Chemistry, vol. 8, no. 3, pp. 301-306, 2006.

[9] O. A. El Seoud, A. Koschella, L. C. Fidale, S. Dorn, and T. Heinze, "Applications of ionic liquids in carbohydrate chemistry: a window of opportunities," Biomacromolecules, vol. 8, no. 9, pp. 2629-2647, 2007.

[10] M. M. Hasani and G. Westman, "New coupling reagents for homogeneous esterification of cellulose," Cellulose, vol. 14, no. 4, pp. 347-356, 2007.

[11] O. Jogunola, V. Eta, M. Hedenström, O. Sundman, T. Salmi, and J.-P. Mikkola, "Ionic liquid mediated technology for synthesis of cellulose acetates using different co-solvents," Carbohydrate Polymers, vol. 135, pp. 341-348, 2016.

[12] A. M. Stepan, A. W. T. King, T. Kakko, G. Toriz, I. Kilpeläinen, and P. Gatenholm, "Fast and highly efficient acetylation of xylans in ionic liquid systems," Cellulose, vol. 20, no. 6, pp. 28132824, 2013.

[13] T. Welton, "Room-temperature ionic liquids. Solvents for synthesis and catalysis," Chemical Reviews, vol. 99, no. 8, pp. 20712083, 1999.

[14] J. D. Holbrey and K. R. Seddon, "Ionic liquids," Clean Products and Processes, vol. 1, no. 4, pp. 223-236, 1999.

[15] L. E. Hamdaoui, M. E. Moussaouiti, and S. Gmouh, "Preparation and characterization of cellulose p-phenylbenzoate by two-step synthesis from microcrystalline and kraft cellulose," Polymer Bulletin, vol. 72, no. 12, pp. 3031-3042, 2015.

[16] M. Earle and K. R. Seddon, in Clean Solvents, M. A. Abraham and L. Moens, Eds., ACS Symposium Series 819, p. 10, American Chemical Society, 2002.

[17] C. M. Gordon, Applied Catalysis A: General, vol. 101, p. 222, 2001.

[18] S. Forsyth, D. R. MacFarlane, R. J. Thomson, and M. von Itzstein, "Rapid, clean, and mild $O$-acetylation of alcohols and carbohydrates in an ionic liquid," Chemical Communications, no. 7, pp. 714-715, 2002.

[19] R. D. Rogers and K. R. Seddon, American Chemical Society, Washington DC, USA, 2002.

[20] P. Wasserscheid and T. Welton, Wiley-VCH, Weinheim, Germany, 2003.

[21] V. W. Goodlett, J. T. Dougherty, and H. W. Patton, "Characterization of cellulose acetates by nuclear magnetic resonance," Journal of Polymer Science Part A: Polymer Chemistry, vol. 9, no. 1, pp. 155-161, 1971.

[22] T. Heinze, K. Schwikal, and S. Barthel, "Ionic liquids as reaction medium in cellulose functionalization," Macromolecular Bioscience, vol. 5, no. 6, pp. 520-525, 2005.

[23] H. Zhang, J. Wu, J. Zhang, and J. He, "1-Allyl-3-methylimidazolium chloride room temperature ionic liquid: a new and powerful nonderivatizing solvent for cellulose," Macromolecules, vol. 38, no. 20, pp. 8272-8277, 2005.

[24] A. P. Abbott, T. J. Bell, S. Handa, and B. Stoddart, "O-Acetylation of cellulose and monosaccharides using a zinc based ionic liquid," Green Chemistry, vol. 7, no. 10, pp. 705-707, 2005.
[25] J. Zhang, J. Wu, Y. Cao, S. Sang, J. Zhang, and J. He, “Synthesis of cellulose benzoates under homogeneous conditions in an ionic liquid," Cellulose, vol. 16, no. 2, pp. 299-308, 2009.

[26] F. Kifani-Sahban, L. Belkbir, and A. Zoulalian, "Study of the slow pyrolysis of Moroccan eucalyptus by thermal analysis," Thermochimica Acta, vol. 284, no. 2, pp. 341-349, 1996.

[27] D. K. Shen and S. Gu, "The mechanism for thermal decomposition of cellulose and its main products," Bioresource Technology, vol. 100, no. 24, pp. 6496-6504, 2009.

[28] P. Rousset, I. Turner, A. Donnot, and P. Perré, “The choice of a low-temperature pyrolysis model at the microscopic level for use in a macroscopic formulation," Annals of Forest Science, vol. 63, no. 2, pp. 213-229, 2006.

[29] H.-P. Fink, P. Weigel, H. J. Purz, and J. Ganster, "Structure formation of regenerated cellulose materials from NMMO-solutions," Progress in Polymer Science, vol. 26, no. 9, pp. 1473-1524, 2001. 

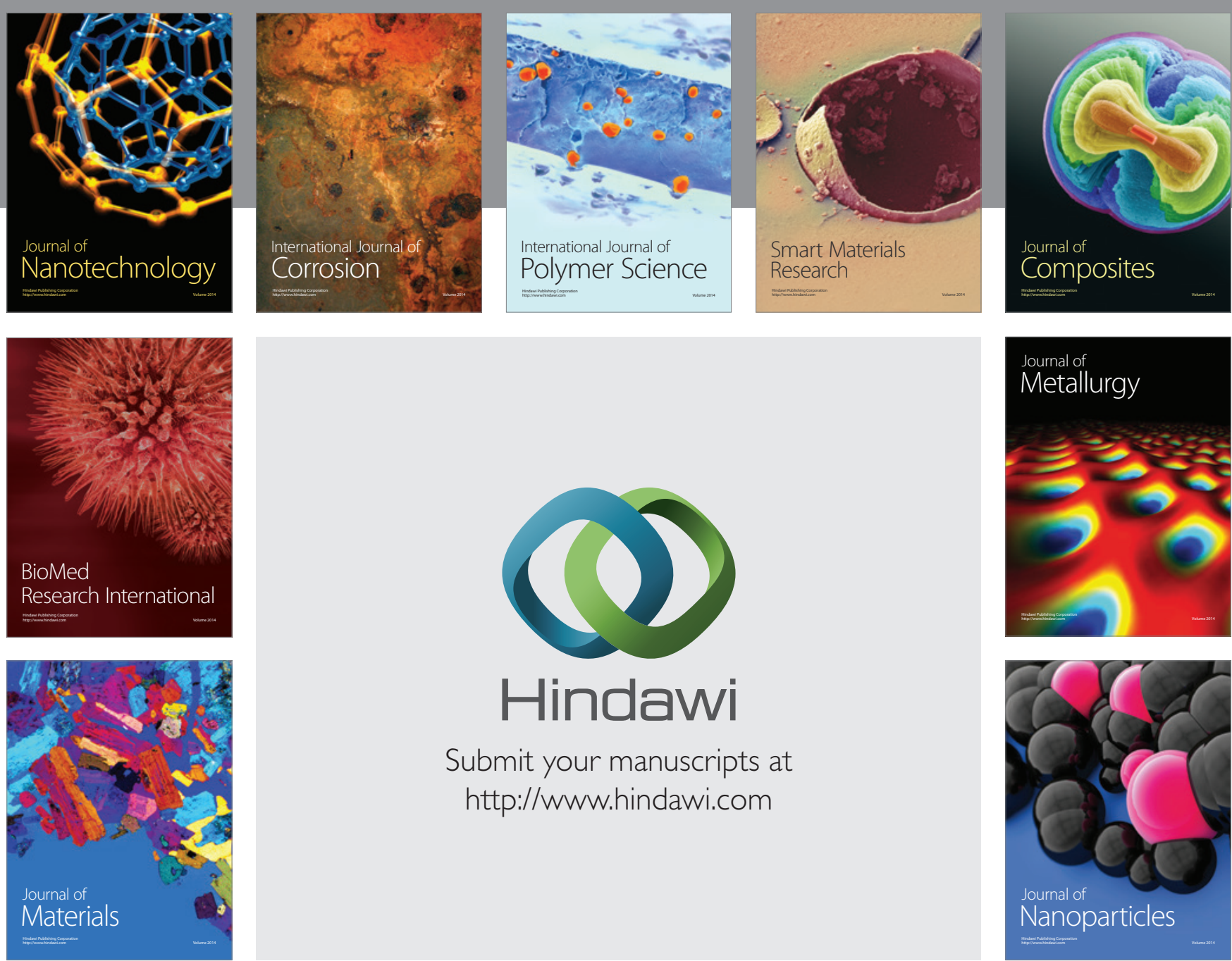

\section{Hindawi}

Submit your manuscripts at

http://www.hindawi.com

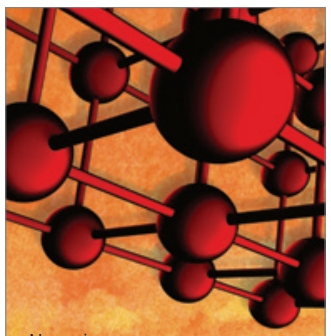

Materials Science and Engineering
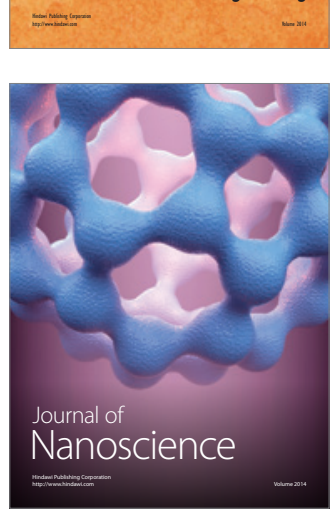
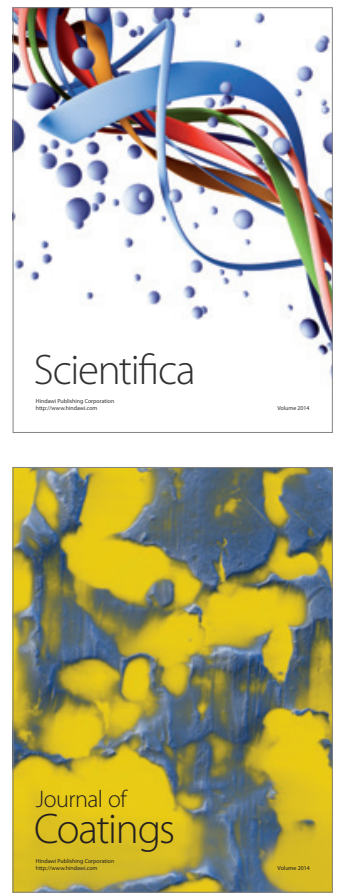
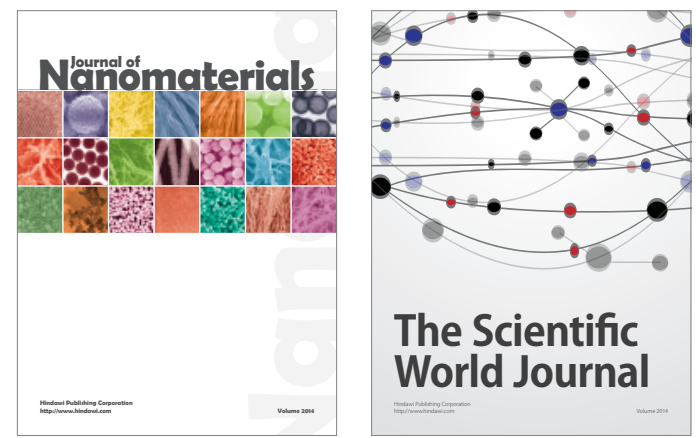

The Scientific World Journal
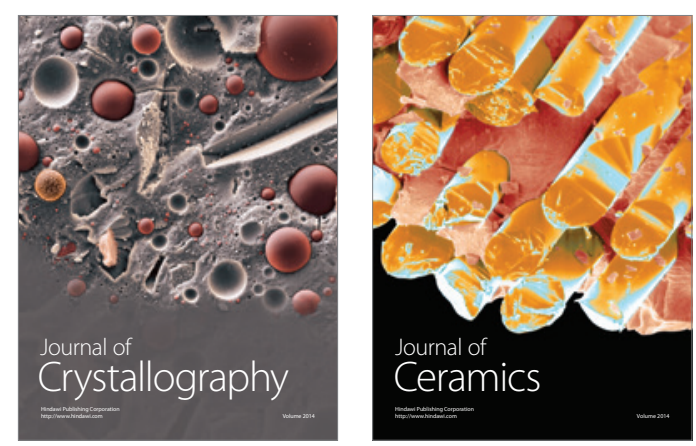
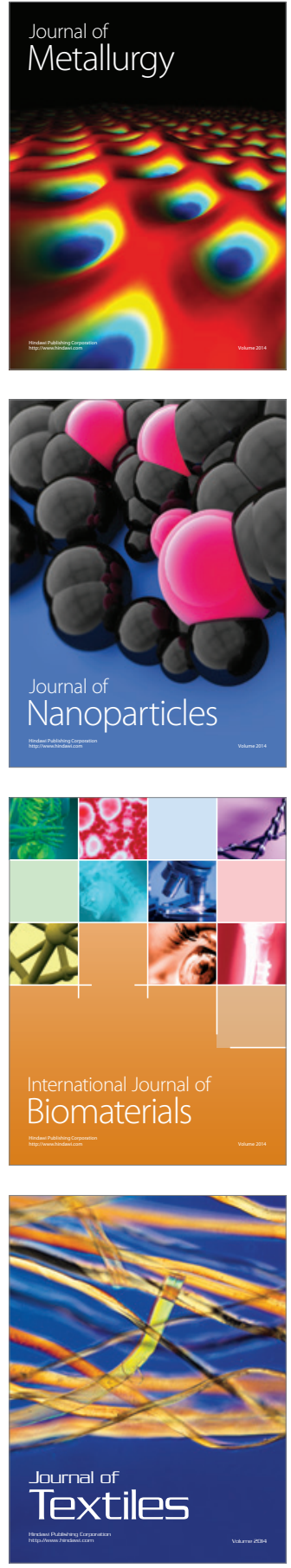